\title{
Silencing of ZNF217 gene influences the biological behavior of a human ovarian cancer cell line
}

\author{
GUIQIN SUN ${ }^{1}$, JUN ZHOU ${ }^{2}$, AILAN YIN ${ }^{1}$, YANQING DING $^{2}$ and MEI ZHONG ${ }^{1}$ \\ ${ }^{1}$ Department of Obstetrics and Gynecology, Nanfang Hospital, ${ }^{2}$ Department of Pathology, \\ Southern Medical University, Guangzhou 510515, Guangdong Province, P.R. China
}

Received January 7, 2008; Accepted February 22, 2008

\begin{abstract}
Zinc-finger protein 217 (ZNF217), a candidate oncogene on 20q13.2, can lead cultured human ovarian and mammary epithelial cells to immortalization, which indicates selective expression of ZNF217 affecting 20q13 amplification during critical early stages of cancer progression. In this study, we tested the hypothesis that ZNF217 is a key factor in regulating ovarian cancer proliferation and progression. We examined the effect of the inhibition of ZNF217 expression on proliferation and invasion by establishing the ZNF217 knockdown ovarian cancer cell line using RNA interference (RNAi). Our results showed that silencing of ZNF217 resulted in the effective inhibition of ovarian cancer cell growth and invasive ability. The results suggested that ZNF217 might play a crucial role in the proliferation and invasion of ovarian cancer.
\end{abstract}

\section{Introduction}

Ovarian cancer causes the highest mortality of the gynecologic cancers; the majority of ovarian cancers are diagnosed in stages III or IV. In these advanced stages, 5-year-survival rate is only $5-25 \%$ after surgery and chemotherapy (1). Ovarian cancer is a heterogeneous disease with respect to histopathology, molecular biology and clinical outcome. In recent studies, the etiology and susceptibility of ovarian cancer showed that the mechanism of carcinogenesis and cancer development is associated with genetic, epigenetic,

Correspondence to: Dr Mei Zhong, Department of Obstetrics and Gynecology, Nanfang Hospital, Southern Medical University, Guangzhou 510515, Guangdong Province, P.R. China

E-mail: zhongmei@fimmu.com

Dr Yanqing Ding, Department of Pathology, Southern Medical University, Guangzhou 510515, Guangdong Province, P.R. China

E-mail: dyq@fimmu.com

Abbreviations: ZNF217, Zinc-finger protein 217; RNAi, RNA interference

Key words: Zinc-finger protein 217, oncogene, RNAi, ovarian cancer, proliferation and environmental factors, but its pathogenesis is still unclear. Most cancer development including ovarian cancer could attribute to an accumulation of genetic and/or epigenetic changes (2).

Zinc-finger protein 217 (ZNF217), recently cloned by positional cloning, is thought as one of the strong candidate oncogenes at $20 \mathrm{q} 13.2$ in breast cancer (3). Its amplification at 20q13.2 has also been frequently found in ovarian cancer and other tumors (4-6) associated with aggressive tumor behavior (7). Additionally, ZNF217 is presumed to encode alternately spliced, Kruppel-like transcription factors of 1,062 and 1,108 aa, each having a DNA-binding domain (eight $\mathrm{C} 2 \mathrm{H} 2$ zinc fingers) and a proline-rich transcription activation domain (3). Furthermore, ZNF217 can be function as a transcriptional repressor and bind to $\mathrm{C}$-terminal binding protein 2 (CtBP2) through a Pro-X-Asp-Leu-Ser (PXDLS) motif and Arg-Arg-Thr (RRT) domain to repress transcription of a variety of genes (8).

In further study of breast cancer, ZNF217 immortalized human mammary epithelial cells (HMEC) when it is overexpressed (9). Similarly, in four independent experiments transducing the gene into finite life span HMEC, ZNF217transduced cultures were found to give rise to immortalized cells (10). In a prostate cancer xenograft, using combined cytogenesis, array-based comparative genomic hybridization (CGH) and expression analyses, ZNF217 was found to be significantly overexpressed in the prostate cancer (6). It is reasonable to assume that an amplicon at $20 \mathrm{q} 13.2$ is likely to harbour one or more putative oncogenes relevant to gastric carcinogenesis, for which ZNF217 is one of the candidates (11).

The frequency of ZNF217 amplification is high in colon cancer and the extent of its amplification varies markedly between tumors (12). Furthermore, poorer survival in patients is associated with either gain or loss of ZNF217. Huang et al (13) also reported that ZNF217 can immortalize HMEC. Additionally, it may play a role in both early- and late-stage breast cancers. In gastric cancer (14), ZNF217 may be associated with specific tumor types or subtypes (8).

It is suggested that $\mathrm{ZNF} 217$, as an ovarian oncogene, is detrimental to senescing normal ovarian surface epithelial cells but contributes to neoplastic progression in ovarian surface epithelial with inactivated p53/RB (15). Moreover, ZNF217 and its associated proteins in a novel pathway may have profound effects on cancer progression (16). 
Table I. Oligonucleotide sequences of ZNF217 specific and negative control siRNA.

\begin{tabular}{ll}
\hline Name & \multicolumn{1}{c}{ Target sequence } \\
\hline pGensil-1/ZNF2171 & GGATGCCTTGTCAATGAAA \\
pGensil-1/ZNF2172 & AAATGTCATCCAAATCGAGGG \\
pGensil-1/Neg & GACTTCATAAGGCGCATGC \\
\hline
\end{tabular}

The role of ZNF217 amplification in ovarian cancer is still unknown. We investigated the mechanism of ZNF217 in regulating ovarian cancer proliferation and invasion in this study by using ZNF217 knockdown ovarian cancer cell line. Our results showed that silencing of ZNF217 resulted in the effective inhibition of ovarian cancer cell growth and the invasive ability.

\section{Materials and methods}

Cell line. The human ovarian cancer cell line ZNF217K D/clone2 was established from metastatic ovarian cancer cell line HO-8910 cells which were obtained from ascites of serous cystadenocarcinoma patients by transfection with pGensil-1/ZNF217 plasmid. ZNF217KD/clone2 cells were cultured in RPMI-1640 medium (Gibco, Grand Island, NY) supplemented with $10 \%$ fetal bovine serum (FBS) at $37^{\circ} \mathrm{C}$ in a humidified atmosphere of $95 \%$ air and $5 \% \mathrm{CO}_{2}$.

Design of shRNA and plasmid preparation. Plasmid vector pGenesil-1 was purchased from Wuhan Genesil Biotechnology Co., Ltd. Two different targeted sequences were designed to be homologous to the ZNF217 mRNA consensus sequence (GeneBank NM_006526.2). A negative control sequence was also designed as the same process, which had no homology with human beings or mice. The complementary oligonucleotides encoded a hairpin structure with a 21-mer stem and a 9-bp loop. The stem was derived from the mRNA target site. The loop sequence separated the two complementary domains. All the sequences were transcribed with DNA polymerase III U6 promoter in plasmid pGensil-1. These two ZNF217 plasmids were named pGensil-1/ ZNF2171 and pGensil-1/ZNF2172 respectively. The negative control plasmid was named pGenesil-1/Neg (Table I). The transformation of these plasmids into competent cells DH5 $\alpha$ and extraction of plasmids followed the routine processes.

Construction of stable silencing lines. Approximately, $1 \times 10^{5}$ cells/well were plated in 24-well plates in medium containing $10 \%$ FBS to grow overnight to $80 \%$ confluency. Transfection of the shRNA oligonucleotides was performed by using Lipofectamine $^{\mathrm{TM}} 2000$ (Invitrogen, USA). HO-8910 cells were divided into blank control group, negative control group and two test groups (ZNF2171 and ZNF2172). Only Lipofectamine $^{\mathrm{TM}} 2000$ was used for transfection in the blank control group. Plasmid pGenesil-1/Neg was used for transfection in the negative control group. Plasmids pGenesil-1/ ZNF2171 and pGenesil-1/ZNF2172 were used for transfection in the test groups (ZNF2171 and ZNF2172) respectively. In the above three groups, the cells were all transfected with the mixture of plasmid and Lipofectamine ${ }^{\mathrm{TM}} 2000$ (1:2) in $100 \mu \mathrm{l}$ serum-free medium. At 6-h post-transfection, $500 \mu \mathrm{l}$ normal medium containing 10\% FBS was added. At $48 \mathrm{~h}$ after cultivation, the medium was replaced by normal medium containing $10 \%$ FBS and $1000 \mu \mathrm{g} / \mathrm{ml} \mathrm{G}-418$ up to selected for stable clones. There were viable cells and discrete G-418 resistance colonies, after 14 days of exposure to G-418.

Real-time reverse transcription polymerase chain reaction (PCR) analysis for ZNF217. cDNA was synthesized by oligo dT primed reverse transcription from $2 \mu \mathrm{g}$ of total RNA using an access reverse transcription system (Promega, Madison, WI). Real-time PCR was performed using Mx3000P RealTime PCR System (Stratagene, La Jolla, CA) and Brilliant SYBR Green QPCR Master Mix kit (Stratagene), following the manufacturer's protocol. Briefly, the reaction mixture (total volume $25 \mu \mathrm{l}$ ) contained $500 \mathrm{ng}$ of cDNA, the forward primer 5'-GAGAAGCGAATGGTGAAAGC-3', and the reverse primer 5'-CAGCGCTCAAGTATGCAAAA-3' to amplify human ZNF217 at a final concentration of $250 \mathrm{nM}$ and with $12.5 \mu 1$ of 2x SYBR Green QPCR Master Mix kit. Thermal cycling conditions were as follows: $94^{\circ} \mathrm{C}$ for $2 \mathrm{~min}$ and 50 cycles at $94^{\circ} \mathrm{C}$ for $30 \mathrm{sec}$, followed by $55^{\circ} \mathrm{C}$ for $30 \mathrm{sec}$ and $72^{\circ} \mathrm{C}$ for $30 \mathrm{sec}$. Experiments were performed in triplicate in the same reaction. Human $\beta$-actin gene was amplified as internal control. Target genes and $\beta$-actin gene were amplified in the same reaction. Comparative quantification is determined using the $2^{-\Delta \Delta C t}$ method (17).

Western blot analysis. Cells were washed twice with cold phosphate-buffered saline (PBS) and lysed on ice in RIPA buffer $1 \mathrm{x}$ PBS, $1 \%$ NP 40, 0.1\% sodium dodecyl sulfate (SDS), $5 \mathrm{mM}$ EDTA, $0.5 \%$ sodium deoxycholate, and $1 \mathrm{mM}$ sodium orthovanadate with protease inhibitors. Protein lysates were resolved on $6 \%$ SDS polyacrylamide gel, electrotransferred to polyvinylidene fluoride membranes (Immobilon P; Millipore, Bedford, MA), and blocked in 5\% non-fat dry milk in Tris-buffered saline, $\mathrm{pH} 7.5(100 \mathrm{mM} \mathrm{NaCl}, 50 \mathrm{mM}$ Tris and $0.1 \%$ Tween-20). Membranes were immunoblotted overnight at $4{ }^{\circ} \mathrm{C}$ with anti-ZNF217 polyclonal antibody (Abcam Biotechnology, USA), and anti-B-actin antibody (Santa Cruz Biotechnology), followed by their respective horseradish peroxidase-conjugated secondary antibodies. Signals were detected by enhanced chemiluminescence (Pierce, Rockford, IL).

In vitro cell growth assay. The cells were prepared at a concentration of $1 \times 10^{4}$ cells $/ \mathrm{ml}$. Aliquots $(100 \mu 1)$ were dispensed into 96-well microtiter plates. The cells were incubated for 1, 2, 3, 4, 5, 6 and 7 days, respectively, and 3(4,5-dimethylthiazol-2-yl)-2,5-diphenyltetrazolium bromide (MTT) assay was performed by adding $20 \mu \mathrm{l}$ of MTT ( $5 \mathrm{mg} / \mathrm{ml}$; Promega) for $4 \mathrm{~h}$. When MTT incubation had been completed, supernatants were removed. Dimethyl sulfoxide (150 ml) (Sigma, St. Louis, MO) was added to each well. Fifteen minutes later, the absorbance value (OD) of each well was measured with a microplate reader set at 570 $\mathrm{nm}$. All experiments were performed in triplicate. 

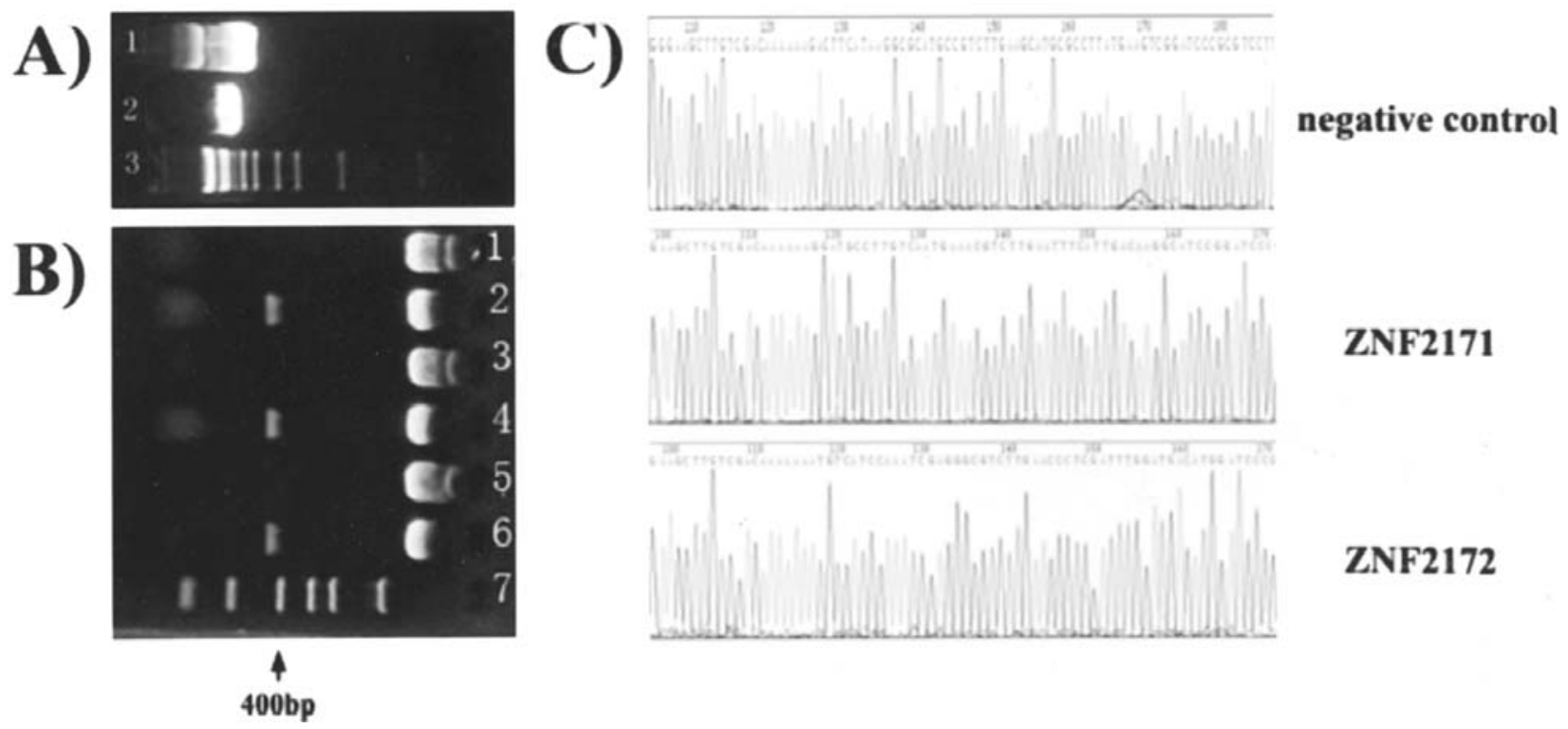

Figure 1. (A) One percent of agarose gel electrophoresis of pGenesil-1 plasmid digested by BamHI and HindIII. 1, pGenesil-1 plasmid; 2, linearization pGenesil-1 plasmid digested by BamHI and HindIII; 3, גEcoT-14 I Marker: 19329 bp, 7743 bp, 6223 bp, 4254 bp, 3472 bp, 2690 bp, 1882 bp, 1489 bp, 925 bp, 421 bp, 74 bp. (B) pGenesil-ZNF217 expression plasmid was identified. 1, ZNF217; 2, ZNF217 SalI; 3, ZNF217; 4, ZNF217 SalI; 5, negative control; 6, negative control SalI; 7, Marker DL2000. (C) Partial sequencing map of plasmid, pGenesil-1/Neg; sequence of recombinant pGenesil-1/ZNF2171 (insertion only); sequence of recombinant pGenesil-1/ZNF2172 (insertion only).

Plate clone formation assay. About $1 \times 10^{2}$ cells/well were plated into a 6-well culture plates, after incubation at $37^{\circ} \mathrm{C}$ for 14 days; the cells were washed twice with PBS and stained with Giemsa solution. The number of colonies containing $\geq 50$ cells was counted under microscope. The plate clone formation efficiency was calculated as: clone formation efficiency $=$ [number of colonies/number of cells inoculated] x $100 \%$.

In vitro invasion assay. This assay was performed using the method of Albini et al (18), with modifications. The cell invasion chamber (Chemicon) contains a polycarbonate membrane with an $8-\mu \mathrm{m}$ pore size, over which a thin layer of ECMatrix (Chemicon) is dried. The extracellular matrix (ECM) layer occludes membrane pores, blocking noninvasive cells from migrating. Warm serum-free medium was added to the top chamber to rehydrate the ECM layer for $2 \mathrm{~h}$ at room temperature. Tumor cells in a serum-free medium ( $300 \mu 1$ containing $1 \times 10^{5}$ cells) were added to the top chamber. The bottom chamber was prepared using $10 \%$ FBS as chemoattactant. After $24 \mathrm{~h}$ of incubation, non-invasive cells were removed with a cotton swab. The cells that had migrated through the membrane and had adhered to the lower surface of the membrane were fixed with methanol and stained with hematoxylin. For quantification, the cells were counted under a microscope in 5 predetermined fields (original magnification, x200).

Statistical analysis. The difference between in vitro cell growths was tested for statistical significance using analysis of variance (ANOVA) for factorial design. Plate clone formation assay and in vitro invasion assay were tested using one-way ANOVA. SPSS 13.0 software (Abbott Laboratories, North Chicago, IL) was used for statistical analysis. $\mathrm{P}<0.05$ was considered statistically significant.

\section{Results}

Construction and validation of plasmid vector. The designing and synthesis of two interfere parts of ZNF217 were compared in GeneBank (www.ncbi.nlm.nih.gov/BLAST/), which were not homologous to other exons. After digestion of pGenesil-1 plasmid by BamHI and HindIII, it was clearly discovered in $1 \%$ agarose gel electrophoresis (Fig. 1A). The multiclone sites of plasmid pGenesil-1 were as follows: HindIII-ShRNA-BamHI-U6 Promotor-EcoRI-SalI-XbaIDraIII. A SalI site for plasmid pGenesil-1 was designed in the inserted fragments between the sites of BamHI and HindIII. If the insertion was correct, a band about $400 \mathrm{bp}$ should be cut-off by SalI. The results of digestion with restriction endonucleases and sequencing showed correct plasmids (Fig. 1B). That was consistent with the plasmids pGenesil-1 and insert shRNA fragments (63 bp) features. The plasmids pGenesil-1/ZNF2171 and pGenesil-1/ ZNF2172 meet the design requirements by digestion. The recombinant plasmid pGenesil-1/ZNF2171, pGenesil-1/ ZNF2172 and pGenesil-1/Neg was sequenced by Wuhan Genesil Biotechnology Co., Ltd. The results showed that the shRNAs, ZNF2171, ZNF2172 were successfully constructed on the pGenesil-1 vector. Sequences were entirely correct (Fig. 1C).

Establishment of ZNF217 silencing ovarian cancer cell line. HO-8910 cells were transduced with pGenesil-1/Neg and pGensil-1/ZNF217. After $24 \mathrm{~h}$ of transduction, recombinant plasmid emitting green fluorescent gathered in cells was observed. As shown in Fig. 2, ZNF2171 vector was most effective in blocking ZNF217 expression. There was no difference in cell morphology. Subsequently, we transduced pGensil-1/ ZNF2171 and pGensil-1/Neg into HO-8910 cells to select G-418-resistant single clones. After 14 days of 


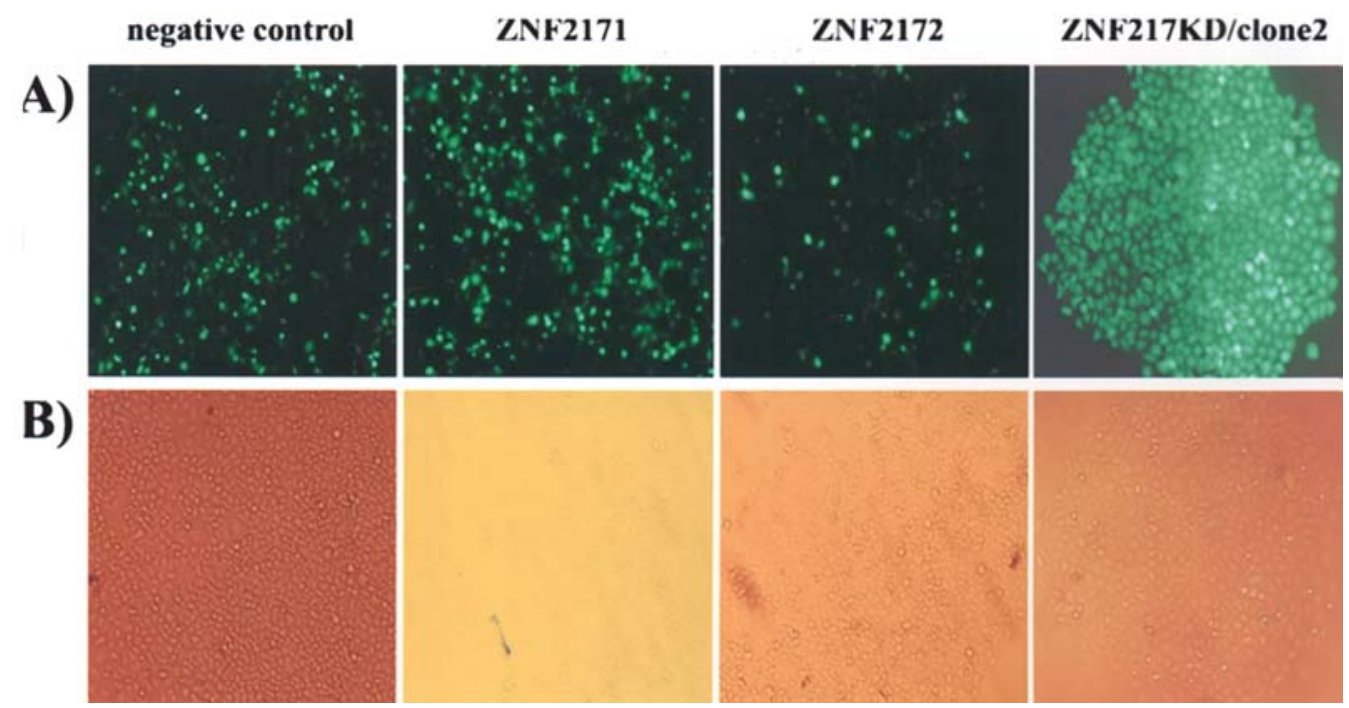

Figure 2. Ovarian cancer cells were transfected by pGenesil-1/ZNF217 plasmid after $24 \mathrm{~h}$ and established neomycin-resistant fluorescent cells (original magnification, x10). (A) Overview in fluorescent microscope. (B) Overview in achromatic light.

A)

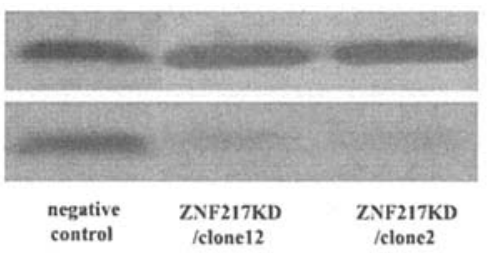

$\beta$-actin

ZNF217

B)

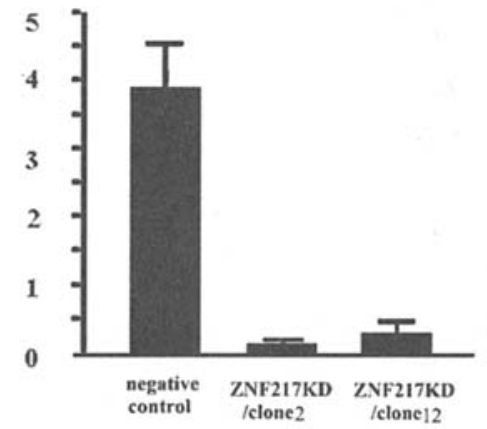

Figure 3. Confirmation of ZNF217 expression in different clones by real-time RT-PCR and Western blot analysis. (A) Western blot analysis shows marked reduction of ZNF217 protein expression in clones 2 and 12; (B) Quantification of ZNF217 mRNA expression in clones transduced by the pGensil-1/ZNF217 plasmid relative to controls (clone transduced by pGensil-1 plasmid).

selection, there were viable cells and discrete G-418 resistance colonies (Fig. 2). After 30 days of selection, transduced clones were expanded and examined by real-time RT-PCR and Western blot analysis for ZNF217 (Fig. 3). Results showed that ZNF217 knock down clone 2 (ZNF217KD/clone 2), knock down clone 12 (ZNF217KD/ clone 12), exhibited 86 and $84 \%$ reduction in ZNF217 protein, respectively. The clone transduced by negative control exhibited no change in ZNF217 expression.
A)

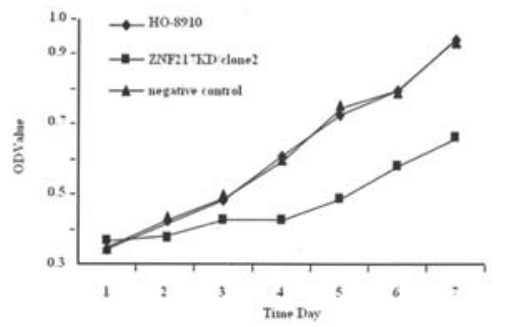

B)

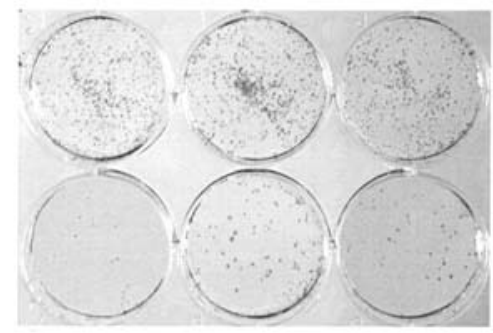

negative control

ZNF217KD/clone2

C)

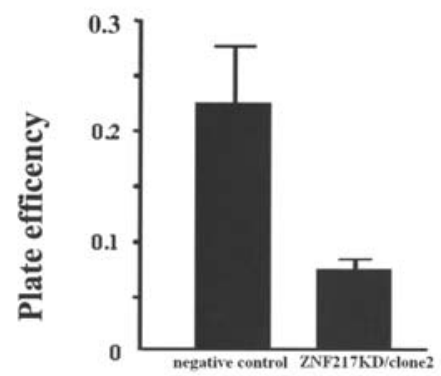

Figure 4. ZNF217 gene silencing suppresses cell proliferation in vitro. (A) The in vitro proliferative abilities of HO-8910 cells, negative control cells, ZNF217KD/clone 2 cells were evaluated by MTT assay. Each value represents the mean $\pm \mathrm{SD}$ of the absorbance value (OD). Results showed that ZNF217 knockdown cells grew significantly more slowly than HO-8910 and negative control cells, and ZNF217 protein expression correlated with cell proliferation. (B and C) The plate colony formation efficiency of negative control cells and ZNF217KD/clone 2 cells. Data represent the mean $\pm \mathrm{SD}$ of triplicate dishes. Compared with negative control cells, ZNF217KD/ clone 2 cells had significantly reduced ability for colony formation $(\mathrm{P}<0.01)$. Results showed that plate colony formation efficiency also correlated with ZNF217 protein expression. 


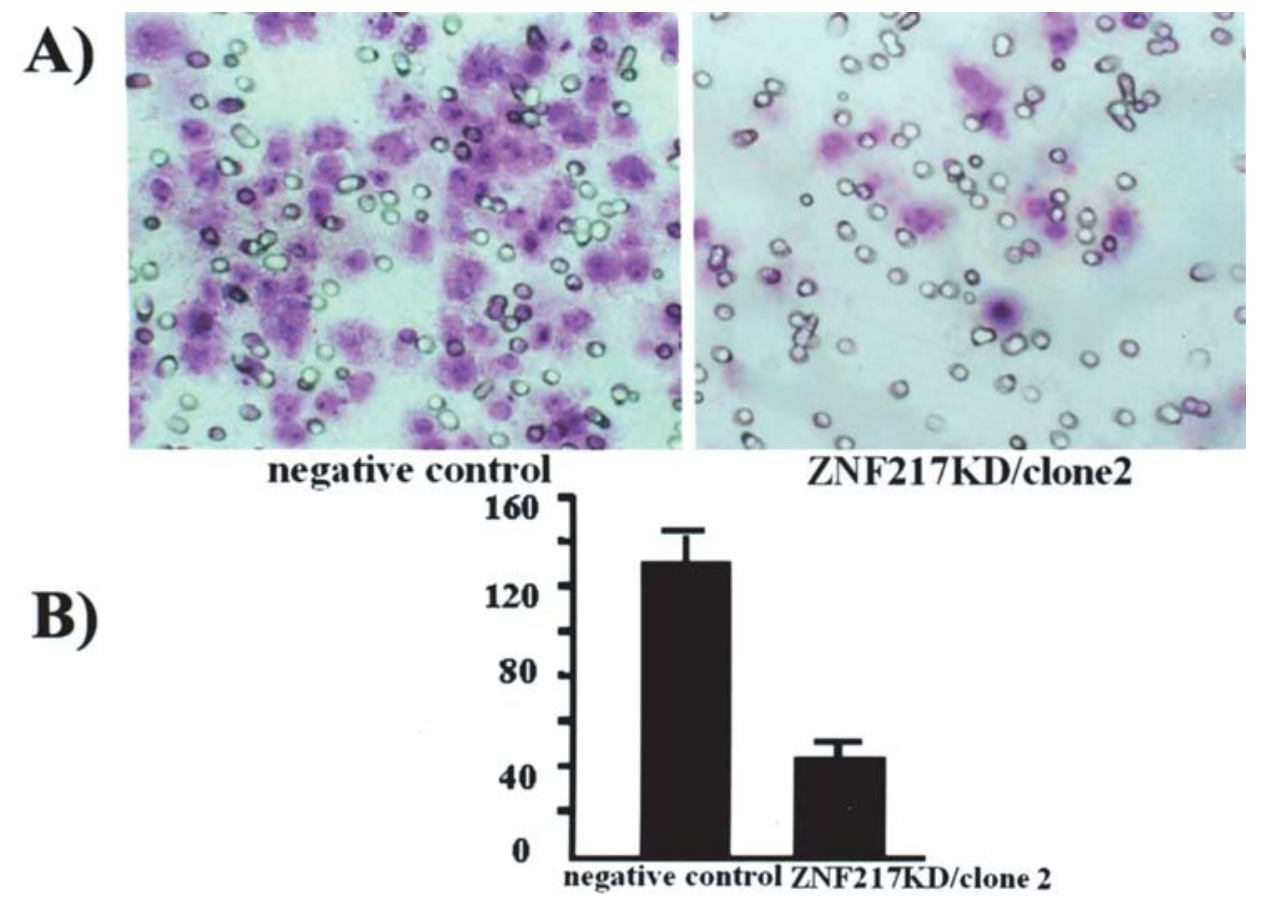

Figure 5. Effect of ZNF217 knockdown on the invasive potential of ovarian cancer cells. In vitro invasion assay was carried out to compare and quantify the invasiveness of negative control cells and ZNF217KD/clone 2. Results are representative of three independent experiments and bars represent the mean \pm SD. ZNF217 silencing cells showed decreased invasion that correlated with ZNF217 protein expression.

ZNF217 gene silencing suppresses cell proliferation in vitro. The effect of ZNF217 protein reduction on the proliferation of ovarian cancer cells was determined by MTT assay and plate clone formation assay. As shown in Fig. 4A, although HO-8910 cells showed in vitro growth ability similar to negative control cells, ZNF217KD/clone 2 had reduced growth ability with respect to HO-8910 cells. Therefore, in vitro cell growth ability was correlated with ZNF217 expression.

The ability of cells to form colonies in plates was examined since there was a correlation between clonogenicity and metastatic capacity. Fig. 4B and C showed that ZNF217 knockdown cells had a significant reduction in their ability to form colonies, compared with negative control cells, and their ability to form colonies correlated with ZNF217 expression.

ZNF217 gene silencing suppresses cell invasion in vitro. The tumor cell invasion through the ECM is an important step in tumor metastasis. ECMatrix serves as a reconstituted basement membrane matrix of proteins. The number of cells migrating to ECMatrix was counted. Compared with negative control cells, ZNF217 knockdown cells decreased invasiveness significantly $(\mathrm{P}<0.01)$, as showed in Fig. 5.

\section{Discussion}

RNAi, was used to introduce of double-stranded RNA into specifically inhibiters of eukaryotic gene expression through the degradation cells to complementary messenger RNA (mRNA). Both siRNA and shRNA are used widely by researchers to silence the expression of many target genes $(19,20)$.
It has been proposed that ZNF217, which is amplified at $20 \mathrm{q} 13$ in various tumor cells, plays a key role during neoplastic transformation. ZNF217 was purified from the complexes that contain repressor proteins such as CtBP2, suggesting that it acts as a transcriptional repressor. However, the function of ZNF217 has not been well characterized due to lack of target genes. Although ZNF217 played a critical role in the $20 \mathrm{q}$ amplification in human breast carcinoma (21), it was amplified less frequently in ovarian carcinomas (22). It was reported that ovarian cancer and breast cancer shared the similar mechanism of carcinogenesis. We found in our previous study, that the amplification of ZNF217 was associated not only with ovarian cancer (23), but also with the tumor stage and poor prognosis of patients with ovarian cancer (24). Like breast cancer, ZNF217 might promote the neoplastic progression of human ovarian surface epithelial cells by introducing important malignancyassociated characteristics, which include immortalization, increasing telomerase activity, stabilization of telomere length, and resistance to transforming growth factor $B$ (TGF$ß)$ growth inhibition (10). Furthermore, ZNF217 likely promotes the neoplastic progression of epithelial ovarian carcinomas (15). However, the exact biological function of ZNF217 is unclear. Therefore, we performed biological characterization of ZNF217 by silencing it in the HO-8910 cell line.

ZNF217 is a typical sequence-specific DNA-binding protein (16). As nuclear transcription factors that regulate the expression of genes, most zinc finger proteins have been found to have nuclear signals. Protein can pass through nuclear pores into the nucleus of the cell (25). Currently, more and more studies have shown that zinc finger protein family members play an important role in development 
process in varieties of cancers (26-28). ZNF217, one of the zinc finger protein family members, was demonstrated to encode alternatively spliced Kruppel-like transcription factors. It has been identified as a new CtBP interaction motif and was established as a transcriptional repressor protein that functions, at least in part, in association with CtBP (8).

Importantly, ZNF217 amplification at 20q13 was associated with aggressive tumor behavior and poor clinical prognosis in breast cancer $(3,7,9)$. In a previous study, we detected ZNF217 overexpression in cell lines and different tissue specimens of ovarian cancer, finding that ZNF217 overexpression was highly related to ovarian cancer $(26,27)$. In the present study, we used a plasmid-mediated RNAi method to obtain an efficient knockdown of ZNF217 gene. Recent results from other laboratories (29) and ours have demonstrated that ZNF217 gene is correlated with cell proliferation and invasion of human ovarian cancer.

In our study, we found that ZNF217 was one of the genes associated with ovarian cancer proliferation. The ability in promoting the proliferation of tumor cells was correlated with invasion and poor outcome (30). We also found that shRNA-mediated knockdown of ZNF217 not only strongly inhibited in vitro cell growth and colony formation efficiency, but reduced their ability to invade ECMatrix-coated membranes in an invasion chamber assay. It was demonstrated that the invasive ability of ovarian cancer cells was correlated with ZNF217 expression, and ZNF217 silencing alone was sufficient to attenuate invasion in ovarian cancer cells. Collectively, data indicated that ZNF217 may be a positive regulator of tumor growth in ovarian cancer, which supports the involvement of ZNF217 in the malignant behavior of human ovarian cancer cells.

The increase in copy numbers and overexpression of ZNF217 in primary tumors, theoretically, may inhibit tumor suppressor gene promoter expression, and enhance tumor cell proliferation, invasion and mobility. The immortalization caused activation of telomerase in tumor cells, leading to tumor growth. The silence of ZNF217 expression in primary human ovarian cancer cell lines significantly inhibited the proliferation, invasion and mobility of ovarian cancer cells. It is suggested that development of ovarian cancer is closely related to the increase of copy numbers and the overexpression of ZNF217 in primary ovarian cancer.

\section{Acknowledgements}

We thank Rangke Wu (Institute of Foreign Language, Southern Medical University, China), Changping Zou (Department of Obstetrics and Gynecology, University of Connecticut, USA) for revising the manuscript.

\section{References}

1. Fehrmann RS, Li XY, van der Zee AG, et al: Profiling studies in ovarian cancer: a review. Oncologist 12: 960-966, 2007.

2. Aunoble B, Sanches R, Didier E and Bignon YJ: Major oncogenes and tumor suppressor genes involved in epithelial ovarian cancer (review). Int J Oncol 16: 567-576, 2000.
3. Collins C, Rommens JM, Kowbel D, et al: Positional cloning of ZNF217 and NABC1: genes amplified at 20q13.2 and overexpressed in breast carcinoma. Proc Natl Acad Sci USA 95: 8703-8708, 1998.

4. Yang SH, Seo MY, Jeong HJ, et al: Gene copy number change events at chromosome 20 and their association with recurrence in gastric cancer patients. Clin Cancer Res 11: 612-620, 2005.

5. Iwabuchi H, Sakamoto M, Sakunaga H, et al: Genetic analysis of benign, low-grade and high-grade ovarian tumors. Cancer Res 55: 6172-6180, 1995

6. Bar-Shira A, Pinthus JH, Rozovsky U, et al: Multiple genes in human 20q13 chromosomal region are involved in an advanced prostate cancer xenograft. Cancer Res 62: 6803-6807, 2002.

7. Tanner MM, Tirkkonen M, Kallioniemi A, et al: Amplification of chromosomal region $20 \mathrm{q} 13$ in invasive breast cancer: prognostic implications. Clin Cancer Res 1: 1455-1461, 1995.

8. Quinlan KG, Nardini M, Verger A, et al: Specific recognition of ZNF217 and other zinc finger proteins at a surface groove of C-terminal binding proteins. Mol Cell Biol 26: 8159-8172, 2006.

9. Collins C, Volik S, Kowbel D, et al: Comprehensive genome sequence analysis of a breast cancer amplicon. Genome Res 11: 1034-1042, 2001.

10. Nonet GH, Stampfer MR, Chin K, Gray JW, Collins CC and Yaswen P: The ZNF217 gene amplified in breast cancers promotes immortalization of human mammary epithelial cells. Cancer Res 61: 1250-1254, 2001.

11. Weiss MM, Snijders AM, Kuipers EJ, et al: Determination of amplicon boundaries at $20 \mathrm{q} 13.2$ in tissue samples of human gastric adenocarcinomas by high-resolution microarray comparative genomic hybridization. J Pathol 200: 320-326, 2003.

12. Rooney PH, Boonsong A, McFadyen MC, et al: The candidate oncogene ZNF217 is frequently amplified in colon cancer. J Pathol 204: 282-288, 2004.

13. Huang G, Krig S, Kowbel D, et al: ZNF217 suppresses cell death associated with chemotherapy and telomere dysfunction. Hum Mol Genet 14: 3219-3225, 2005.

14. Zhu YQ, Zhu ZG, Liu BY, Chen XH, Zhang Y and Yin HR: Study on amplification of ZNF217 in primary gastric carcinoma. Zhonghua Wei Chang Wai Ke Za Zhi 8: 60-62, 2005.

15. Li P, Maines-Bandiera S, Kuo WL, et al: Multiple roles of the candidate oncogene ZNF217 in ovarian epithelial neoplastic progression. Int J Cancer 120: 1863-1873, 2007.

16. Cowger JJ, Zhao Q, Isovic $M$ and Torchia J: Biochemical characterization of the zinc-finger protein 217 transcriptional repressor complex: identification of a ZNF217 consensus recognition sequence. Oncogene 26: 3378-3386, 2007.

17. Livak KJ and Schmittgen TD: Analysis of relative gene expression data using real-time quantitative PCR and the 2(-Delta Delta C(T)) Method. Methods 25: 402-408, 2001.

18. Albini A, Iwamoto Y, Kleinman HK, et al: A rapid in vitro assay for quantitating the invasive potential of tumor cells. Cancer Res 47: 3239-3245, 1987.

19. Gartel AL and Kandel ES: RNA interference in cancer. Biomol Eng 23: 17-34, 2006.

20. Lu PY, Xie F and Woodle MC: In vivo application of RNA interference: from functional genomics to therapeutics. Adv Genet 54: 117-142, 2005.

21. Tanner MM, Tirkkonen M, Kallioniemi A, et al: Independent amplification and frequent co-amplification of three non-syntenic regions on the long arm of chromosome 20 in human breast cancer. Cancer Res 56: 3441-3445, 1996.

22. Tanner MM, Grenman S, Koul A, et al: Frequent amplification of chromosomal region 20q12-q13 in ovarian cancer. Clin Cancer Res 6: 1833-1839, 2000.

23. Zhong M, Li J, Ding YQ and Song LL: ZNF217 gene was detected in ovarian serous cystadenocarcinoma by fluorescence in situ hybridization. Zhonghua Yi Xue Yi Chuan Xue Za Zhi 23: 665-667, 2006.

24. Li J, Zhong M, Song LL and Su GD: Oncogene ZNF217 amplification on chromosome $20 \mathrm{q}$ in ovarian serous cystadenocarcinoma and its clinical implications. Nan Fang Yi Ke Da Xue Xue Bao 26: 824-825, 2006.

25. Yano K, Ueki N, Oda T, Seki N, Masuho Y and Muramatsu M: Identification and characterization of human ZNF274 cDNA, which encodes a novel kruppel-type zinc-finger protein having nucleolar targeting ability. Genomics 65: 75-80, 2000. 
26. Odeberg J, Rosok O, Gudmundsson GH, et al: Cloning and characterization of ZNF189, a novel human Kruppel-like zinc finger gene localized to chromosome 9q22-q31. Genomics 50: 213-221, 1998.

27. Nawrath M, Pavlovic J and Moelling K: Inhibition of human hematopoietic tumor formation by targeting a repressor MybKRAB to DNA. Cancer Gene Ther 7: 963-972, 2000.

28. Hajra KM, Chen DY and Fearon ER: The SLUG zinc-finger protein represses E-cadherin in breast cancer. Cancer Res 62: 1613-1618, 2002.
29. Suzuki S, Egami K, Sasajima K, et al: Comparative study between DNA copy number aberrations determined by quantitative microsatellite analysis and clinical outcome in patients with stomach cancer. Clin Cancer Res 10: 3013-3019, 2004.

30. Whitfield ML, George LK, Grant GD and Perou CM: Common markers of proliferation. Nat Rev Cancer 6: 99-106, 2006. 